University of Nebraska - Lincoln

DigitalCommons@University of Nebraska - Lincoln

2010

Semiclassical complex-time method for tunneling ionization: Molecular suppression and orientational dependence

Gordon A. Gallup

University of Nebraska-Lincoln, ggallup1@unl.edu

Ilya I. Fabrikant

University of Nebraska-Lincoln, ifabrikant@unl.edu

Follow this and additional works at: https://digitalcommons.unl.edu/physicsgallup

Part of the Physics Commons

Gallup, Gordon A. and Fabrikant, llya I., "Semiclassical complex-time method for tunneling ionization: Molecular suppression and orientational dependence" (2010). Gordon Gallup Publications. 54.

https://digitalcommons.unl.edu/physicsgallup/54

This Article is brought to you for free and open access by the Research Papers in Physics and Astronomy at DigitalCommons@University of Nebraska - Lincoln. It has been accepted for inclusion in Gordon Gallup Publications by an authorized administrator of DigitalCommons@University of Nebraska - Lincoln. 


\title{
Semiclassical complex-time method for tunneling ionization: Molecular suppression and orientational dependence
}

\author{
Gordon A. Gallup and Ilya I. Fabrikant \\ Department of Physics and Astronomy, University of Nebraska, Lincoln, Nebraska 68588-0111, USA
}

(Received 28 December 2009; published 22 March 2010)

\begin{abstract}
We apply a previously developed semiclassical complex time method to the calculation of tunneling ionization of several diatomic molecules and $\mathrm{CO}_{2}$. We investigate the presence or absence of the molecular suppression effect by calculating ionization rates of $\mathrm{N}_{2}$ versus $\mathrm{Ar}, \mathrm{O}_{2}$ versus $\mathrm{Xe}, \mathrm{F}_{2}$ versus $\mathrm{Ar}$, and $\mathrm{CO}$ versus $\mathrm{Kr}$. Comparisons with other theories, including the molecular-orbital-Ammosov-Delone-Krainov (MO-ADK) model and the strong-field approximation, are given. We also analyze the dependence of the ionization rate on the angle $\theta_{F}$ between the molecular axis and the field direction. The theoretical results agree quite well with experiment for $\mathrm{N}_{2}$ and $\mathrm{O}_{2}$ but give too low a value of the peak angle $\theta_{F}$ for $\mathrm{CO}_{2}$. Our calculations give small values of the ionization rates for $\mathrm{O}_{2}$ and $\mathrm{CO}_{2}$ at $\theta_{F}=0$ and $90^{\circ}$, in agreement with experiment. Other calculations, including the MO-ADK model and methods involving a numerical integration of the time-dependent Schrödinger equation, exhibit substantially weaker suppression at these angles.
\end{abstract}

DOI: 10.1103/PhysRevA.81.033417

PACS number(s): $32.80 . R m, 33.80 . R v, 32.60 .+\mathrm{i}$

\section{INTRODUCTION}

Tunneling ionization of atoms and molecules is an important mechanism of laser ionization at low frequencies, or small Keldysh parameters (a.u.) [1]

$$
\gamma=\omega \frac{\sqrt{2 I_{p}}}{F_{0}},
$$

where $\omega$ is the frequency of the ac field, $I_{p}$ is the ionization potential, and $F_{0}$ is the electric field amplitude. Tunneling ionization is a first step in several strong-field phenomena such as high-order harmonic generation, multi-electron ionization, and, more generally, in all phenomena where the rescattering process plays a role [2].

Most of the methods for the calculation of tunneling ionization can be assigned to one of three categories. The first class of methods is based on the quasiclassical asymptotic theory developed by Smirnov and Chibisov [3] and Perelomov et al. [4]. Later it became known as ADK (Ammosov-DeloneKrainov) theory. This term is somewhat misleading because the ADK article [5] just uses the results that were obtained 20 years earlier in Refs. [3,4]. However, since this term has become so common, and because the ADK generalization for molecules developed by Tong et al. [6] was dubbed the MO-ADK, we will continue using this terminology below. In addition to the low-frequency approximation, corresponding to small $\gamma$, ADK theory assumes that the regions of the electron interaction with atom and the electron interaction with the external field can be spatially separated. As a result, this method is, strictly speaking, valid for relatively weak fields. On the other hand, for weak fields the Keldysh parameter $\gamma$ becomes large, therefore the ADK method is often extended into the region of strong fields and even into the region where the classical ionization becomes possible, called the barrier-suppression region, although the use of ADK theory does not make sense at all if there is no tunneling. Several attempts [7-9] have been made to extend the static-field ionization model to the barrier-suppression region.
The second class of methods is based on the Keldysh theory [1] and is related to the strong-field approximation (SFA). This theory treats the ionization process as a transition between an atomic (or molecular) state and the "dressed" (by the electron-field interaction) final state. It can be applied to the field of any frequency, in particular, it allows us to obtain the tunneling limit (for small values of $\gamma$ ). But this theory neglects the final-state electron interaction with the atomic or molecular residue, therefore it can be applied only for sufficiently strong fields. It also cannot describe rescattering which is important in the strong-field regime. Several calculations [10-15] using these methods have been performed recently for ionization of diatomic molecules.

The third class of methods is based on numerical integration of the time-dependent Schrödinger equation (TDSE). These methods are very challenging computationally, particularly for ionization of molecules. Several calculations [16-18] have been available for some time for the simplest molecular system, $\mathrm{H}_{2}^{+}$. More recently, results for diatomic molecules $\mathrm{N}_{2}, \mathrm{O}_{2}$, and $\mathrm{F}_{2}$ [19-21] and triatomic $\mathrm{CO}_{2}$ [22,23] were obtained.

It is clear that for complex molecules methods of intermediate complexity should be developed, bridging simple analytical results, like those from MO-ADK formulas, and more complicated methods like those using the TDSE. The recently developed semiclassical approach [24] is based on an obvious observation: all the complexity of the quantummechanical many-electron problem is contained in the inner region of the configuration space, when the ionized electron is close to the remainder of the system. When the electron is well separated from the molecule, its motion becomes simple and can be described by quasiclassical or semiclassical methods. This idea was used by Brabec et al. [25] for description of tunneling ionization of complex molecules. We have chosen a somewhat different complex time technique $[4,26]$ which is based on the analytical continuation of the classical equations of motion in the complex time plane. The first applications of this method [24] to rare-gas atoms and the simplest molecular targets, $\mathrm{H}_{2}^{+}$and $\mathrm{H}_{2}$, were aimed to check the validity of the 
MO-ADK theory. They show that the ADK and MO-ADK results usually overestimate the ionization rate by a factor of 2 to 3 , but the ionization rate ratios for two systems agree quite well.

This observation is important for an explanation of the phenomenon of "molecular suppression" [27-29], that is, a reduction of the ionization rate in a molecule as compared to that of a "companion" atom with the same or close ionization potential. Our analysis of the simplest case of molecular suppression, $\mathrm{H}_{2}$ versus $\mathrm{Ar}$, gives practically the same results as the MO-ADK theory of Tong et al. [6]. However, there are some other examples that are more challenging to the theory. In particular, ionization of the $F_{2}$ molecule was predicted to be substantially suppressed by both MO-ADK [6] theory and the interference model of Muth-Böhm et al. [30]. However, the subsequent experiment [29] did not confirm this prediction. Two recent calculations [20,31] show that there is no suppression in $\mathrm{F}_{2}$; however, the mechanism for this is under dispute. Whereas Telnov and Chu [20,21] claim that the total ionization probability is enhanced due to substantial contribution of inner molecular orbitals, Usachenko et al. [31] claim that this contribution is completely negligible and explain ionization enhancement by strong electron correlation in $F_{2}$. (See also Sec. IV.)

Another issue that recently received attention from both experimental and theoretical communities is the dependence of the ionization rate on the molecular orientation, since the ionization of oriented molecules by intense low-frequency fields can be a tool for imaging electron densities [18]. Dependence of the ionization rate on molecular orientation relative to the direction of the ionizing field was measured for $\mathrm{CO}$ [32], $\mathrm{N}_{2}, \mathrm{O}_{2}$, and $\mathrm{CO}_{2}[33,34]$ molecules. Several calculations of the dependence of ionization rates on molecular orientation were performed in the framework of MO-ADK theory [35-37]. The most challenging case is $\mathrm{CO}_{2}$ for which measurements of Pavičić et al. [34] produce a much narrower distribution than that given by the MO-ADK theory. Two recent TDSE calculations [22,23] did not resolve this puzzle.

In the present article we apply our semiclassical propagation method [24] to analysis of several cases of molecular suppression. We also address the problem of the angular dependence of ionization rates and compare our results for $\mathrm{N}_{2}, \mathrm{O}_{2}$, and $\mathrm{CO}_{2}$ with MO-ADK calculations and experimental results.

Atomic units are used throughout the article. We will be using two frames of reference: the body frame where the molecular axis is directed along the $z$ axis and the external field lies in the $x z$ plane and the field frame with the field directed along the negative $z$ axis, so the force $\mathbf{F}$ on electron is along the positive $z$ axis. For simplicity, by the field direction we will mean the direction of the force $\mathbf{F}$.

\section{SEMICLASSICAL METHOD}

\section{A. Outline}

The rate of tunneling ionization of a molecule can be calculated by integrating the tunneling current density $J$ over a plane perpendicular to the electric field (which in the field frame is directed along the negative $z$ axis) [24]

$$
\Gamma=\int J(\rho) \rho d \rho d \phi
$$

In the semiclassical complex-time method the current density at $z \rightarrow \infty$ is obtained by calculating complex classical trajectories emerging perpendicular to a spherical surface of radius $r_{0}$ surrounding the molecule. If the initial coordinates on the surface are given by $\mathbf{q}$, and the quantum-mechanical wave function on the surface is $\left.\psi_{(} \mathbf{q}\right)$, the current density is given by

$$
J=\operatorname{Im}\left(\psi^{*} \frac{d \psi}{d z}\right)=|\psi(\mathbf{q}) A(\mathbf{r}, \mathbf{q})|^{2} e^{-2 \operatorname{Im} S} \frac{d \operatorname{Re} S}{d z},
$$

where $S(\mathbf{r}, \mathbf{q})$ is the reduced action corresponding to the trajectory going from $\mathbf{q}$ to $\mathbf{r}$ and the semiclassical amplitude

$$
A(\mathbf{r}, \mathbf{q})=\sqrt{\left|J_{t \rightarrow 0}(\mathbf{r}, \mathbf{q}) / J_{t}(\mathbf{r}, \mathbf{q})\right|}
$$

with [38]

$$
J_{t}(\mathbf{r}, \mathbf{q})=\partial[\mathbf{r}(t)] / \partial(t, \mathbf{q}) .
$$

This Jacobian probes the dependence of a trajectory endpoint $\mathbf{r}(t)$ on the time-of-flight $t$ and on the coordinates $\mathbf{q}$ of the starting point on the initial surface.

The initial wave function $\psi(\mathbf{q})$ is calculated taking account of the electric field using standard quantum chemistry codes.

\section{B. Angular distrbution}

The major contribution to the integral (2) is given by $\rho=0$ since the exponential in the integral decays fast with $\rho$, unless the energy of the electron is close to the top of the potential barrier. Therefore the ionization rate is proportional to $\mid \psi(\theta=$ $0)\left.\right|^{2}$, where $\theta$ is the escape angle relative to the field axis. We conclude that the dependence of the ionization rate on the angle between molecular axis and the field direction is given by the angular dependence of the molecular probability density.

This conclusion was made before within the framework of the MO-ADK theory [35] and was also confirmed by direct integration of TDSE for $\mathrm{H}_{2}^{+}$[18]. Since $\psi(\mathbf{q})$ is a polarized orbital, the present approach allows us to study how the angular distribution depends on the electric field. In the body frame the dependence of the ionization rate on the angle $\theta_{F}$ between the molecular axis and the field direction is given by

$$
\Gamma=\text { const } \cdot\left|\psi\left(F, \theta_{F}, \theta_{P}\right)\right|^{2}, \quad \theta_{P}=\theta_{F},
$$

where $\theta_{P}$ is the spherical angle of the electron radius-vector in the body frame. In this approximation $\Gamma$ goes to zero whenever the field $\mathbf{F}$ is directed along the nodal plane for a given molecular orbital. For example, for a $\pi_{g}$ orbital in a linear molecule (like $\mathrm{O}_{2}$ or $\mathrm{CO}_{2}$ ) the probability density is 0 at $\theta_{P}=0$ and $\theta_{P}=90^{\circ}$. In the presence of an arbitrarily directed external field cylindrical symmetry is lost, but the Hamiltonian still preserves the symmetry with respect to reflection in the plane containing the molecular axis and the electric field. The orbitals are classified according to irreducible representation of the $C_{s}$ group. As a result, we obtain two orbitals, $A^{\prime}$ (symmetric) and $A^{\prime \prime}$ (antisymmetric), which can also be approximately classified as $\pi_{x}$ and $\pi_{y}$, if we keep the field vector $\mathbf{F}$ in the $z x$ plane. According to Eq. (6) the orbital $\pi_{y}$ does not contribute 
to the ionization rate at all, since it is zero on the symmetry plane. The contribution of the orbital $\pi_{x}$ is 0 at $\theta_{P}=0$ and $\theta_{P}=90^{\circ}$, since at $\theta_{F}=0$ the orbital becomes antisymmetric with respect to the transformation $x \rightarrow-x$, and at $\theta_{F}=90^{\circ}$ antisymmetric with respect to the transformation $z \rightarrow-z$.

However, the discussed effect results from the approximate treatment of integration in Eq. (2): it is assumed that the relatively slowly varying function $|\psi|^{2}$ can be taken at the value corresponding to the zero escape angle and factored out. Strictly speaking, if $\psi$ goes to 0 in the direction of the field, other escape angles should be included. In the field frame the direction of the field is given by $\theta=0$. If $\psi$ goes to 0 in this direction, then

$$
\psi(\theta) \approx\left(\frac{d \psi}{d \theta}\right)_{\theta=0} \theta
$$

and Eqs. (2) and (3) become

$$
\Gamma=\left(\frac{d \psi}{d \theta}\right)_{\theta=0}^{2} \int|\theta(\rho) A(\mathbf{r}, \mathbf{q})|^{2} e^{-2 \operatorname{Im} S} \frac{d \operatorname{Re} S}{d z} \rho d \rho d \phi,
$$

where $\theta(\rho)$ is the complex escape angle as a function of the cylindrical coordinate $\rho$ of the trajectory end point.

\section{Contribution of the $\pi_{y}$ orbital}

A similar approach can be used to estimate the contribution of the $\pi_{y}$ orbital for an arbitrary field direction. In this case the orbital is 0 at the azimuthal angle $\phi_{P}=0$ in the body frame, and in parallel with Eq. (7) we can write

$$
\psi \approx\left(\frac{d \psi}{d \phi_{P}}\right)_{\phi_{P}=0} \phi_{P}(\rho, \phi)
$$

where $\phi_{P}(\rho, \phi)$ is the azimuthal angle in the body frame as a function of $\rho$ and $\phi$, the azimuthal angle in the field frame. Before we proceed with the integration, we should express $\phi_{P}$ in terms of the angles $\theta$ and $\phi$ in the field frame.

The transformation from the body frame to the field frame is given by the matrix

$$
M=\left(\begin{array}{ccc}
\cos \theta_{F} & 0 & \sin \theta_{F} \\
0 & 1 & 0 \\
-\sin \theta_{F} & 0 & \cos \theta_{F}
\end{array}\right),
$$

where $\theta_{F}$ is the angle between the field and the molecular axis. This allows us to write the required transformation as

$$
\begin{gathered}
\sin \theta_{P} \cos \phi_{P}=\sin \theta \cos \phi \cos \theta_{F}+\cos \theta \sin \theta_{F} \\
\sin \theta_{P} \sin \phi_{P}=\sin \theta \sin \phi \\
\cos \theta_{P}=-\sin \theta \cos \phi \sin \theta_{F}+\cos \theta \cos \theta_{F} .
\end{gathered}
$$

We are interested in the limits as $\theta$ and $\phi_{P} \rightarrow 0$, thus obtaining

$$
\phi_{P} \approx \theta \frac{\sin \phi}{\sin \theta_{P}} \approx \theta \frac{\sin \phi}{\sin \theta_{F}+\theta \cos \theta_{F} \cos \phi} .
$$

If we ignore the unimportant case $\theta_{F}=0$, then

$$
\phi_{P} \approx \theta \frac{\sin \phi}{\sin \theta_{F}} \text {. }
$$

We note that $\phi_{P}$ should be considered as an analytical continuation since the escape angle $\theta$ is complex.
Eqs. (2) and (3) can now be written as

$$
\Gamma=\int\left(\frac{d \psi}{d \phi_{P}}\right)_{\phi_{P}=0}^{2}\left|\phi_{P}(\rho, \phi) A(\mathbf{r}, \mathbf{q})\right|^{2} e^{-2 \operatorname{Im} S} \frac{d \operatorname{Re} S}{d z} \rho d \rho d \phi,
$$

where the dependence of $\phi_{P}$ on $\rho$ and $\phi$ is given by Eq. (13) and the dependence of $\theta$ on $\rho$. The derivative $d \psi / d \phi_{P}$ is a function of $\theta_{P}$, and therefore a function of $\rho$ and $\phi$ as well. However, since the major contribution to the integral is given by escape angles close to 0 , we can assume that $\theta_{P}=\theta_{F}$. Therefore the derivative is independent of integration variables and can be factored out. The $\phi$ integration then becomes trivial

$$
\int \sin ^{2} \phi d \phi=\pi
$$

and the integration over $\rho$ is performed numerically as before.

\section{Occupation number dependence}

Most of the theories of tunneling ionization use the oneelectron approximation and do not discuss the dependence of the ionization rate on the orbital occupation number, although some authors suggest to multiply the rates by the highest-occupied-molecular-orbital (HOMO) occupancy [11]. Because of the strong dependence of the tunneling ionization rate on the binding energy, usually only ionization of the HOMO is calculated, although it was claimed recently $[19,20]$ that in some cases ionization of the HOMO-1 can make a substantial contribution. Even if we restrict our theory to ionization of the HOMO, there is still the question of how the ionization rate depends on its occupation number. In the Appendix we show that if the total wave function of the outer shell can be described by a Slater determinant with one orbital, the result for one-electron ionization rate should be multiplied by its occupation number. Consider, for example, the important cases of $\mathrm{O}_{2}$ and $\mathrm{F}_{2}$ molecules. In the absence of the field their outer-shell electronic configurations are $\pi_{g}^{2}$ and $\pi_{g}^{4}$, respectively. In the presence of the field they become $\pi_{x} \pi_{y}$ and $\pi_{x}^{2} \pi_{y}^{2}$. Accordingly, for $\mathrm{F}_{2}$ the ionization rate for the $\pi_{x}$ and $\pi_{y}$ orbitals should be multiplied by two, which partly explains the absence of the molecular suppression effect in $\mathrm{F}_{2}$ compared to that in $\mathrm{O}_{2}$. We note, however, that this effect is purely probabalistic. As was suggested by Usachenko et al. [31], there are also dynamical effects in $\mathrm{F}_{2}$ which further increase its ionization rate.

\section{E. Comparison with the MO-ADK theory}

A preliminary comparison with the MO-ADK theory [24] showed that for simple systems like $\mathrm{H}_{2}^{+}$and $\mathrm{H}_{2}$, it somewhat overestimates the rate, but the ratios of the molecular ionization rate to the ionization rate for a companion atom with the same ionization potential agree quite well with the semiclassical theory.

Turning to more complex targets, we should proceed with caution. First, the version of the MO-ADK theory developed in Ref. [6] does not incorporate the occupation number dependence of the ionization rate, and when we are making comparisons, we should make sure that occupation numbers are included in both rates. Second, disagreement might be 


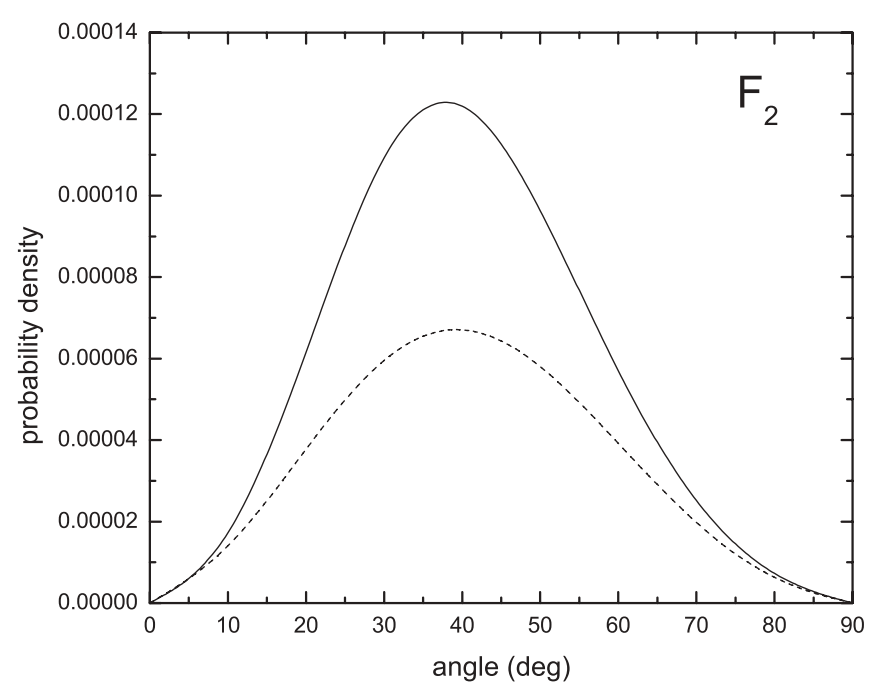

FIG. 1. The probability density for the $\mathrm{F}_{2}\left(\pi_{g}\right)$ orbital at $r=5$ a.u.; a comparison of the present results (solid line) with multiplescattering method, Ref. [6](dashed line).

due to two reasons: basic approximations made in MO-ADK theory as discussed in Ref. [24] and different molecular orbitals employed in calculations. In their first calculations, Tong et al. $[6,35]$ used the multiple-scattering method to find molecular orbitals, which were then expanded in spherical harmonics. Asymptotic coefficients required by the MO-ADK theory were then calculated from the resulting radial wave functions. As was pointed out in Refs. [11,12] and later in Ref. [37], the resulting coefficients suffer from significant inaccuracies that were corrected for in $\mathrm{CO}_{2}$ [37]. To demonstrate the difference between the orbitals used in Ref. [6] and those employed in our calculations, in Fig. 1 we present comparisons of $\pi_{x}$ probability densities $\left|\psi\left(r, \theta_{P}, \phi_{P}\right)\right|^{2}$ for the $\mathrm{F}_{2}$ molecule for $r=5$ a.u., $\phi_{P}=0$, and $F=0$. Although the shapes agree qualitatively quite well, the multiple-scattering approach significantly underestimates the probability density. To give an idea of how this inaccuracy affects the ionization rates published in Ref. [6], in Table I we present the ratios of angle-averaged probability densities for several molecules. The entry for $\mathrm{N}_{2}$ is consistent with the correction obtained by Kjeldsen and Madsen [11]. For comparison purposes, we will use two versions of the MO-ADK theory, the original, with asymptotic coefficients published in Ref. [6], and an "adjusted" version with the coefficients multiplied by factors presented in Table I.

TABLE I. The ratio of angle-averaged probability density calculated in the present work to that of Ref. [6] at $r=5$ a.u.

\begin{tabular}{lcc}
\hline \hline Molecule & Symmetry & Ratio \\
\hline $\mathrm{N}_{2}$ & $\sigma_{g}$ & 3.12 \\
$\mathrm{O}_{2}$ & $\pi_{x}$ & 1.50 \\
$\mathrm{~F}_{2}$ & $\pi_{x}$ & 2.58 \\
$\mathrm{CO}$ & $\sigma$ & 5.93 \\
$\mathrm{NO}$ & $\pi$ & 1.65 \\
\hline \hline
\end{tabular}

\section{F. Molecular orbital calculations}

All of the quantum chemical calculations for this study were performed in the same way as in Ref. [24], using the GAMESS [39] and CRUNCH [40] routines and the finite field method to determine the polarized orbitals. Gaussian bases were required for $\mathrm{C}, \mathrm{N}, \mathrm{O}$, and $\mathrm{F}$, and these were the standard 6-311G $(s, p, d)$ versions provided by the built-in data in GAMESS. These bases produced polarizabilities and anisotropies within $85-95 \%$ of experiment using either the induced dipole or the energy change method. All the orbitals were determined with the $a b$ initio spin-restricted Hartree-Fock or spin-restricted openshell Hartree-Fock method.

\section{RESULTS AND DISCUSSION}

\section{A. Field dependence of ionization rate}

In this section our goal is to analyze absolute values of the ionization rates and their dependence on the field and on the molecular orientation. For randomly oriented molecules we average our result over the angle between the molecular axis and the field direction. We will assume that molecular vibrations do not affect the ionization process and will use the vertical ionization potential in our calculations. Although some vibrational effects were found in ionization of $\mathrm{H}_{2}$ [12,41], in the molecules of primary interest to us, like $\mathrm{N}_{2}, \mathrm{O}_{2}, \mathrm{CO}$, these effects are small [12]. We will also address the problem of "molecular suppression," that is, we compare the ionization rate for molecules with those for companion atoms having the same ionization potential. In most cases we will present the static rate $\Gamma_{s t}$ as a function of the field amplitude $F$ in a.u.. As is well known [4,5], the dynamical rate $\Gamma_{\text {dyn }}$ can be obtained from the static by the equation

$$
\Gamma_{\text {dyn }}=\sqrt{\frac{3 F}{\pi \kappa^{3}}} \Gamma_{s t},
$$

where $\kappa=\sqrt{2 I_{p}}$ and $I_{p}$ is the ionization potential. The static field can be converted into the laser peak intensity $I_{0}$ by $I_{0}=$ $(F / 0.05338)^{2}$, where $I_{0}$ is the peak intensity in $10^{14} \mathrm{~W} / \mathrm{cm}^{2}$.

In Fig. 2 we present the ionization rate for randomly oriented $\mathrm{N}_{2}$ molecules and compare them with the original and adjusted versions of the MO-ADK theory. Apparently the agreement between the present semiclassical calculations and the "original" MO-ADK theory is fortuitous due to difference in the probability densities, as indicated in Table I. The "adjusted" MO-ADK result is about a factor of 3 higher that is consistent with the previous comparisons.

In Fig. 3 we compare the case of randomly oriented $\mathrm{N}_{2}$ molecules with that of molecules oriented along the field. To address the issue of molecular suppression, we also present the ionization rates for Ar. The $\mathrm{N}_{2}$ rates are "suppressed" only for randomly oriented molecules. For aligned molecules the rates are actually higher than those for Ar. This result can be easily explained by analysis of the angular dependence of the ionization rate (see the next subsection). Since the experiments [28,42] on the ionization rate for $\mathrm{N}_{2}$ versus $\mathrm{Ar}$ are not conclusive about the molecular suppression, one can assume that a partial alignment could play a role.

Our results for aligned molecules agree quite well with recent calculations of Usachenko et al. [31] performed in the 


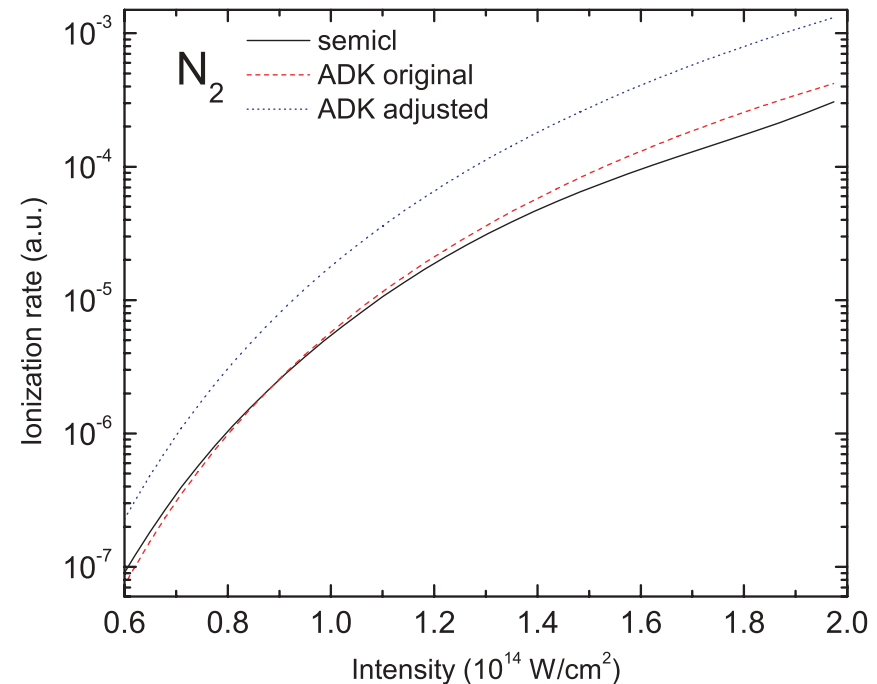

FIG. 2. (Color online) The dynamical ionization rate as a function of intensity for the $\mathrm{N}_{2}$ molecule. Comparison of the present results with the original and adjusted versions of the MO-ADK theory.

velocity-gauge SFA combined with a density-functional theory (DFT) for calculating molecular orbitals. The agreement suggests that the tunneling approximation (or approximation assuming a small Keldysh parameter) is justified in the present case. For the intensity $I=10^{14} \mathrm{~W} / \mathrm{cm}^{2}$ of Ti:sapphire laser radiation, the original Keldysh parameter, Eq. (1), is of the order of 1 . Note that the Keldysh parameter represents the ratio of the tunneling time to the laser period. However, the tunneling time in the original Keldysh parameter, Eq. (1), corresponds to motion in a short-range (strictly speaking, zero-range) potential and does not take into account the influence of the Coulomb field. In complex molecules the tunneling time can be significantly smaller, which leads to a substantial reduction of the effective Keldysh parameter [43].

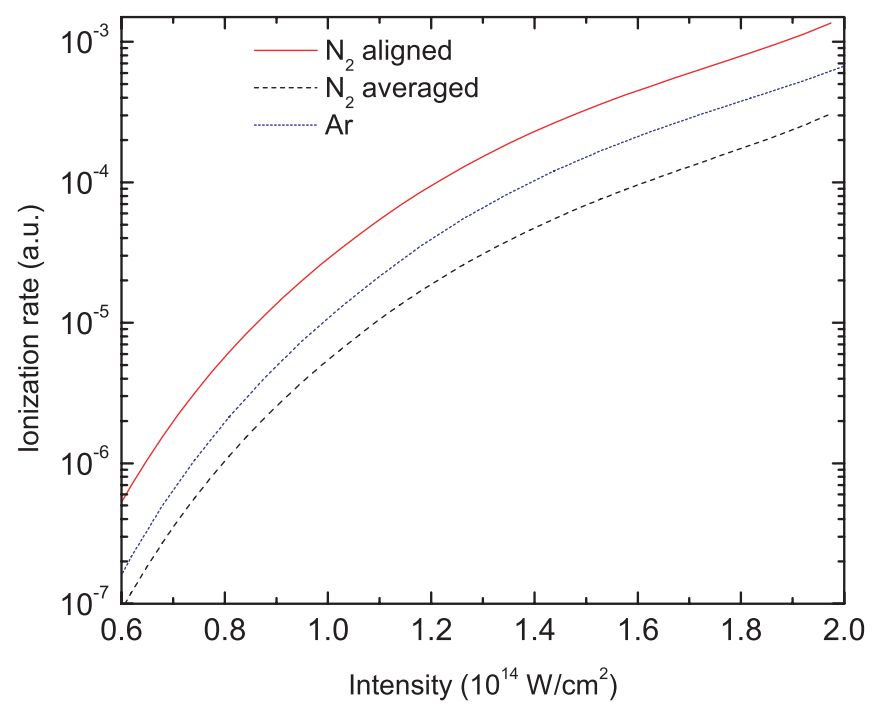

FIG. 3. (Color online) The dynamical ionization rate as a function of intensity for $\mathrm{N}_{2}$ versus Ar. Results for $\mathrm{N}_{2}$ are given for randomly oriented molecules and molecules aligned along the field.

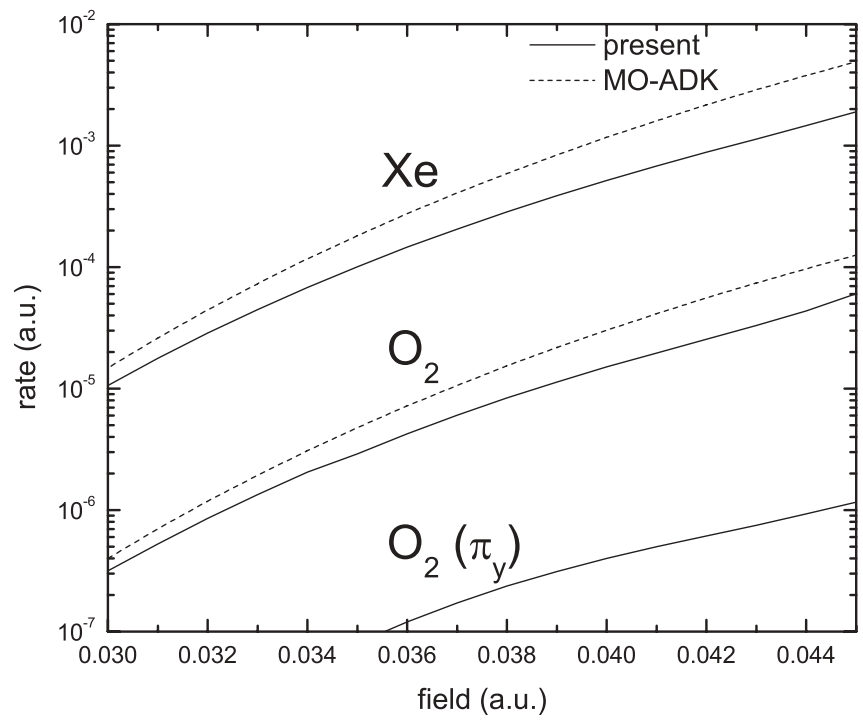

FIG. 4. The static ionization rate as a function of electric field for $\mathrm{O}_{2}$ versus Xe. Comparison with MO-ADK theory (dashed lines).

Telnov and Chu [21] found a substantial contribution of the HOMO-1 orbital $\left(1 \pi_{u}\right)$ to the ionization of $\mathrm{N}_{2}$. They explain this by the resonance between HOMO and HOMO1 in the 800-nm laser field. The tunneling theory cannot, of course, incorporate this effect. However, calculations of Usachenko et al. [31], which were based on the Keldysh theory describing both tunneling and multiphoton regimes, show that the contribution of HOMO-1 is insignificant. This issue remains to be resolved by future calculations and experiments.

In Fig. 4 we compare the ionization rate for $\mathrm{O}_{2}$ with those for Xe and with the "original" MO-ADK rates. Since the ionization potential of the $\mathrm{O}_{2}$ molecule is rather low, the threshold value $F_{\text {th }}$ for the above-barrier ionization, where the present theory breaks down, is low too, $F_{\text {th }}=0.0489$ a.u., which corresponds to a threshold intensity $I_{\text {th }}=0.838 \times$ $10^{14} \mathrm{~W} / \mathrm{cm}^{2}$. Therefore we present our calculation in the region below $F=0.046$ a.u., where our results are somewhat lower than the original MO-ADK calculations. Adjustment increases the difference further. Both theories, when compared with the companion, $\mathrm{Xe}$, demonstrate a strong molecular suppression, confirming experimental results [27,28]. We also present the $\pi_{y}$ contribution which is typically only about $2 \%$ of the total rate in this field range.

In Fig. 5 we compare ionization rates for $\mathrm{F}_{2}$ and Ar. Both semiclassical and MO-ADK results include the occupation number factor in the ionization rate, but the MO-ADK rates are not adjusted with regard to the probability density. The present theory still gives a suppression of the $F_{2}$ rates as compared to that of Ar, but this suppression, about a factor 2.5 , is not as big as was previously obtained in the MO-ADK [6] and interference models [30]. This conclusion agrees with the results of Telnov and Chu [21], who found for the suppression factor 4.2 and 1.9 for the intensities $2 \times$ $10^{14} \mathrm{~W} / \mathrm{cm}^{2}$ and $5 \times 10^{14} \mathrm{~W} / \mathrm{cm}^{2}$, respectively. Note, however, that these factors were obtained for molecules oriented at $40^{\circ}$ with respect to the field direction. Since this orientation provides the maximum ionization rate, the suppression factors will be somewhat higher for randomly oriented molecules. 


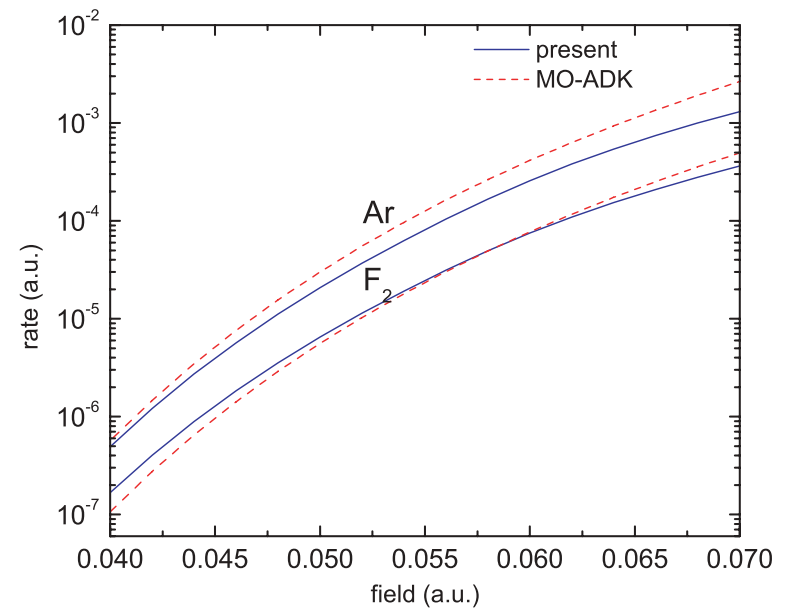

FIG. 5. (Color online) The static ionization rate as a function of electric field for $\mathrm{F}_{2}$ versus Ar. Comparison with MO-ADK theory.

Alignment along the field leads to a further suppression of the ionization probability for $\mathrm{F}_{2}$ since the $\pi_{g}$ orbital is 0 on the molecular axis. It is therefore unclear why the ionization rates of Usachenko et al. [31] for $\mathrm{O}_{2}$ and $\mathrm{F}_{2}$ are not strongly suppressed, since, according to the caption to Fig. 3 of their article, their calculation corresponds to "strongly aligned" molecules. Usachenko et al. explain the absence of suppression in $F_{2}$ by a substantially more enhanced and pronounced electron-correlation response to the laser field as compared to the $\mathrm{O}_{2}$ case. This suggests that inclusion of electron correlation in our model might further improve agreement with the experiment on ionization of $\mathrm{F}_{2}$ compared with $\mathrm{Ar}$ [42] which demonstrates no suppresssion.

In Fig. 6 we compare ionization rates for $\mathrm{CO}$ and $\mathrm{Kr}$. The original MO-ADK calculations give substantially lower rates because of the strongly underestimated probability density; see Table I. Our results show almost no suppression, in agreement with existing experiments [29].

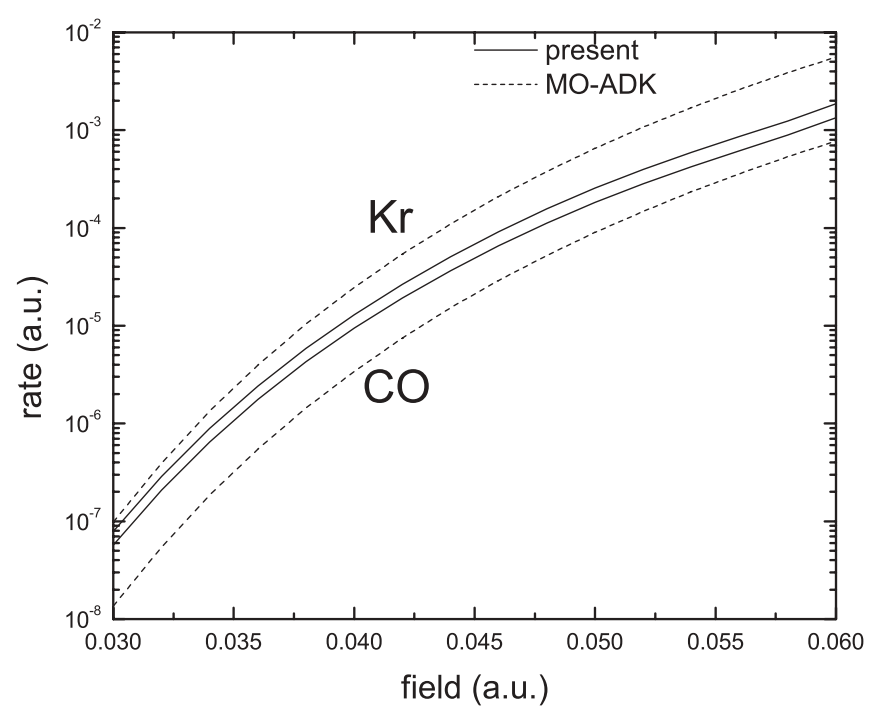

FIG. 6. The static ionization rate as a function of electric field for $\mathrm{CO}$ versus $\mathrm{Kr}$. Comparison with MO-ADK theory.

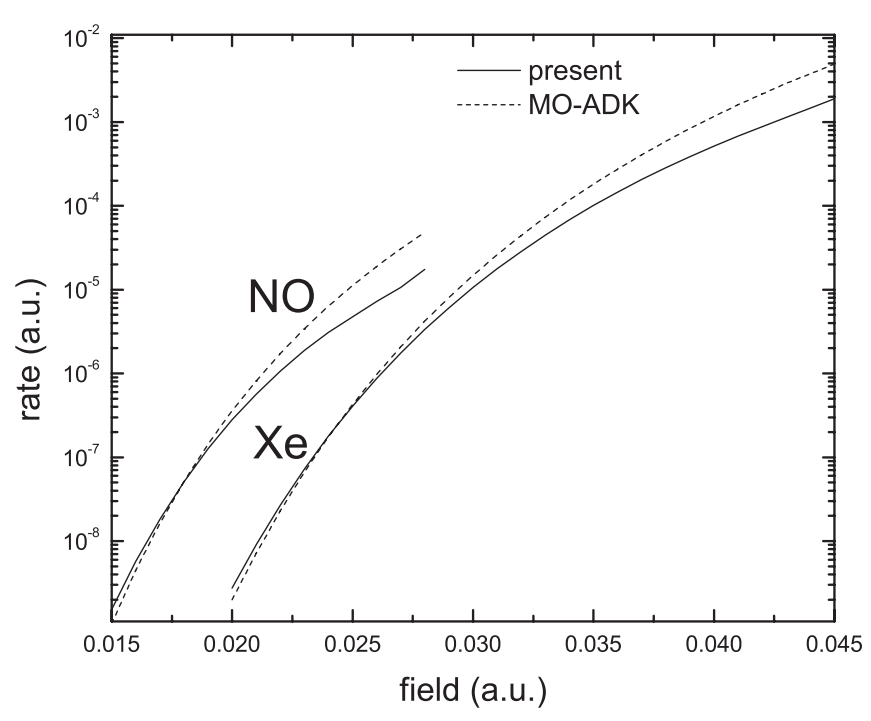

FIG. 7. The static ionization rate as a function of electric field for $\mathrm{NO}$ versus Xe. Comparison with MO-ADK theory.

In Fig. 7 we present ionization rates for NO molecules. The ionization potential for $\mathrm{NO}, 9.26 \mathrm{eV}$, is relatively low, and the corresponding threshold for the above-barrier ionization is $F_{\text {th }}=0.029$ a.u.. Since there is no companion atom for $\mathrm{NO}$, we compare ionization rates with $\mathrm{Xe}\left(I_{p}=12.13 \mathrm{eV}\right)$, as was suggested by Tong et al. [6]. In this case both theories agree quite well except in the near-threshold region. As was shown by Tong et al., MO-ADK theory agrees very well with measurements except in the region of weak fields where the Keldysh parameter becomes too large.

\section{B. Angular dependence}

In this subsection we discuss the dependence of the ionization rate on $\theta_{F}$, the angle between the field and the molecular axis. To compare angular dependencies for different fields (or intensities), we present an arbitrarily normalized function $\Gamma\left(\theta_{F}\right)$ for each field. All molecules considered below are linear with inversion symmetry, therefore only the range of $\theta_{F}$ from 0 to $90^{\circ}$ is plotted in each case. With regard to the triatomic $\mathrm{CO}_{2}$ one might suggest that if the molecule were not aligned along the field, it could be bent by the field and the inversion symmetry be broken. However, the molecular geometry does not change significantly during the fast ionization process. Therefore we assume that the molecule is in the field a short-enough time so that ionization occurs from the field-free nuclear configuration.

To compare the present theory with experimental data, we will sometimes present calculations for intensities above those corresponding to the above-barrier ionization threshold. Although, strictly speaking, our theory is not applicable in this field range, we assume that the angular distribution does not change drastically when we go above the threshold.

In Fig. 8 we present the angular dependence for the $\mathrm{N}_{2}$ molecule and compare it with the experiment of Pavičić et al. [34]. The distribution changes significantly when the intensity is growing from 0 to $3.1 \times 10^{14} \mathrm{~W} / \mathrm{cm}^{2}$. In contrast, the MO-ADK distributions presented in Fig. 9 [44] show virtually no change with intensity in this intensity range. This can be 


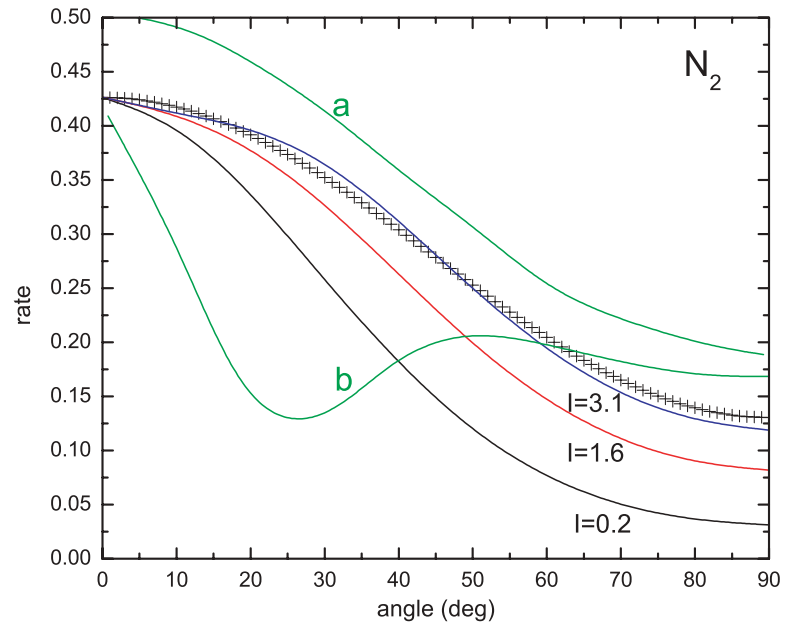

FIG. 8. (Color online) The $\mathrm{N}_{2}$ ionization rate as a function of angle $\theta_{F}$ between the field direction and the molecular axis, normalized to 0.4263 at $\theta_{F}=0$, for three values of intensity given in units of $10^{14} \mathrm{~W} / \mathrm{cm}^{2}$. Curves a and $\mathrm{b}$, total ionization probability and the HOMO contribution for $I=2 \times 10^{14} \mathrm{~W} / \mathrm{cm}^{2}$ calculated by Telnov and Chu [21]. (Note that curves ' $a$ ' and 'b' are normalized arbitrarily, but the relative values are given as in Ref. [21].) Crosses, experiment [34] at $I=1.5 \times 10^{14} \mathrm{~W} / \mathrm{cm}^{2}$.

easily understood by noticing that the field dependence of the angular distribution in the MO-ADK theory appears due to the contribution of states with $|m|>0$, which is very small in case of $\sigma_{g}$ orbital in $\mathrm{N}_{2}$. In contrast, the present theory involves a polarized orbital whose shape changes significantly with the field.

In the same figure we also present comparison with time-dependent density-functional calculations of Telnov and Chu [21], who found a substantial contribution of the HOMO-1 orbital to the ionization probability. Although the shape of the curve describing the contribution of HOMO (curve b) differs substanitally from ours, the total ionization

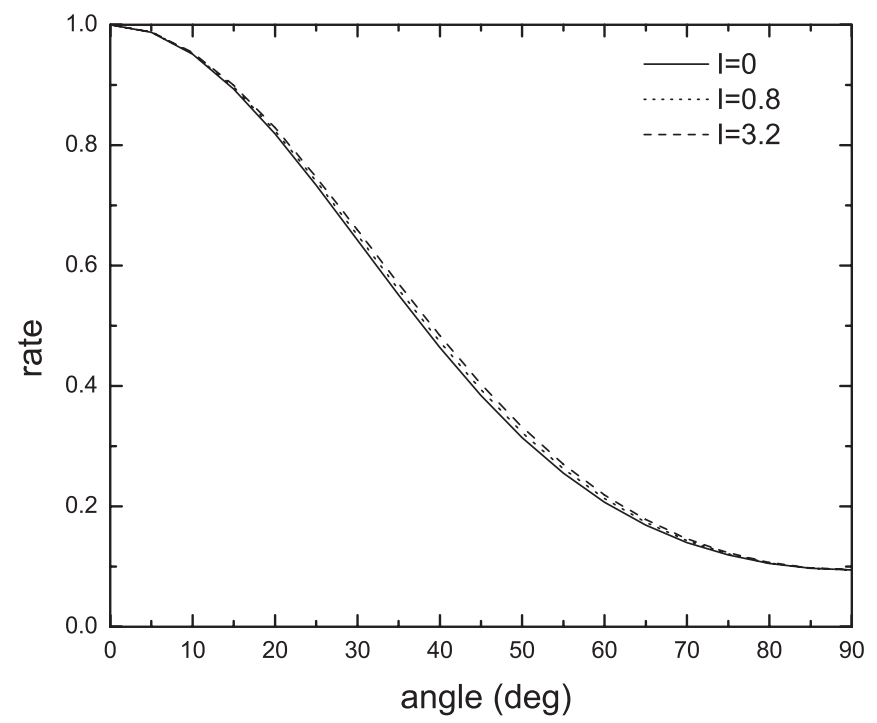

FIG. 9. The $\mathrm{N}_{2}$ ionization rate as a function of $\theta_{F}$ calculated according to the MO-ADK theory, normalized to 1 at $\theta_{F}=0$. Field intensity is given in units $10^{14} \mathrm{~W} / \mathrm{cm}^{2}$.

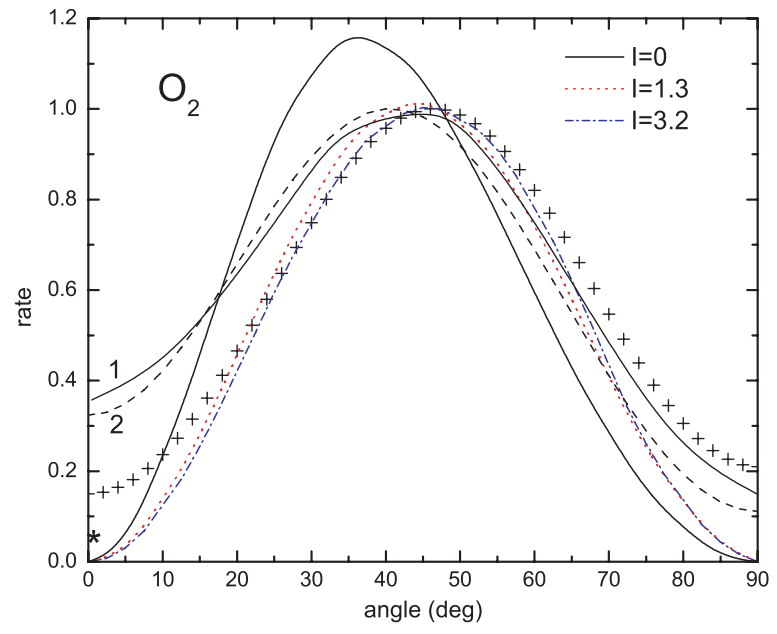

FIG. 10. (Color online) The $\mathrm{O}_{2}$ ionization rate as a function of $\theta_{F}$, normalized to 1 at $\theta_{F}=47.5^{\circ}$, for three values of intensity, given in $10^{14} \mathrm{~W} / \mathrm{cm}^{2}$. The asterik (*) at zero angle is the estimate calculated according to Eq. (15). Other theories: solid curve 1, TDSE results of Telnov and Chu [20] for $I=1 \times 10^{14} \mathrm{~W} / \mathrm{cm}^{2}$. Dashed curve 2, MO-ADK results for $I=1.3 \times 10^{14} \mathrm{~W} / \mathrm{cm}^{2}$. Both are normalized to 1 at the peak. Crosses, experiment [34] at $I=1.3 \times 10^{14} \mathrm{~W} / \mathrm{cm}^{2}$.

probability (curve a) exhibits the angular dependence which is practically the same as the present result. This agreement looks fortuitous to us.

Our result for $I=1.6 \times 10^{14} \mathrm{~W} / \mathrm{cm}^{2}$ is close, but somewhat lower at larger angles, to measurements of Pavičić et al. [34] at $I=1.5 \times 10^{14} \mathrm{~W} / \mathrm{cm}^{2}$. We note that the above-barrier threshold intensity in this case is $I_{\mathrm{th}}=2.36 \times 10^{14} \mathrm{~W} / \mathrm{cm}^{2}$.

In Fig. 10 we present the angular distribution for $\mathrm{O}_{2}$. We note that in contrast to $\mathrm{MO}-\mathrm{ADK}$ calculations presented in the same figure, our rate goes to 0 at $\theta_{F}=0$ and $90^{\circ}$ because of the symmetry property of the $\pi_{x}$ orbital. As was discussed in subsection II B, these 0 values are approximate. If we include complex trajectories with nonzero escape angles, the ionization rate $\Gamma$ is given by Eq. (14). If we normalize $\Gamma\left(\theta_{F}\right)$ to 1 at $\theta_{F}=\theta_{0}$, then

$$
\Gamma(0)=\frac{1}{\left|\psi\left(\theta_{0}\right)\right|^{2}}\left(\frac{d \psi}{d \theta}\right)_{\theta=0}^{2} \frac{\int|\theta(\rho) B(\mathbf{r})|^{2} \rho d \rho}{\int|B(\mathbf{r}, \mathbf{q})|^{2} \rho d \rho}
$$

where

$$
B(\mathbf{r}, \mathbf{q})=|A(\mathbf{r}, \mathbf{q})|^{2} e^{-2 \operatorname{Im} S} \frac{d \operatorname{Re} S}{d z} .
$$

An estimate for $\Gamma(0)$ is presented in Fig. 10. It is substantially lower than the experimentally observed signal for $\theta_{F}=0$, but overall agreement with the experiment is very good, particularly in view of the fact that the above-barrier ionization threshold in this case is $I_{\text {th }}=0.84 \times 10^{14} \mathrm{~W} / \mathrm{cm}^{2}$ because of a low ionization potential for $\mathrm{O}_{2}$. The peak value of $\theta_{F}$ moves from $36^{\circ}$ at low intensities to $45^{\circ}$ at $I=1.3 \times 10^{14} \mathrm{~W} / \mathrm{cm}^{2}$. In contrast, the MO-ADK peak angle is almost independent of intensity and stays at $\theta_{F}=41^{\circ}$. Another distinguished feature of the MO-ADK result is that it gives a large contribution at $\theta_{F}=0$ and $90^{\circ}$. Although the SFA calculations [10] also give nonzero results at these angles, they are substantially lower than the MO-ADK result. Moreover, Jaron-Becker et al. [10] emphasize that with an increasing field the orientation 


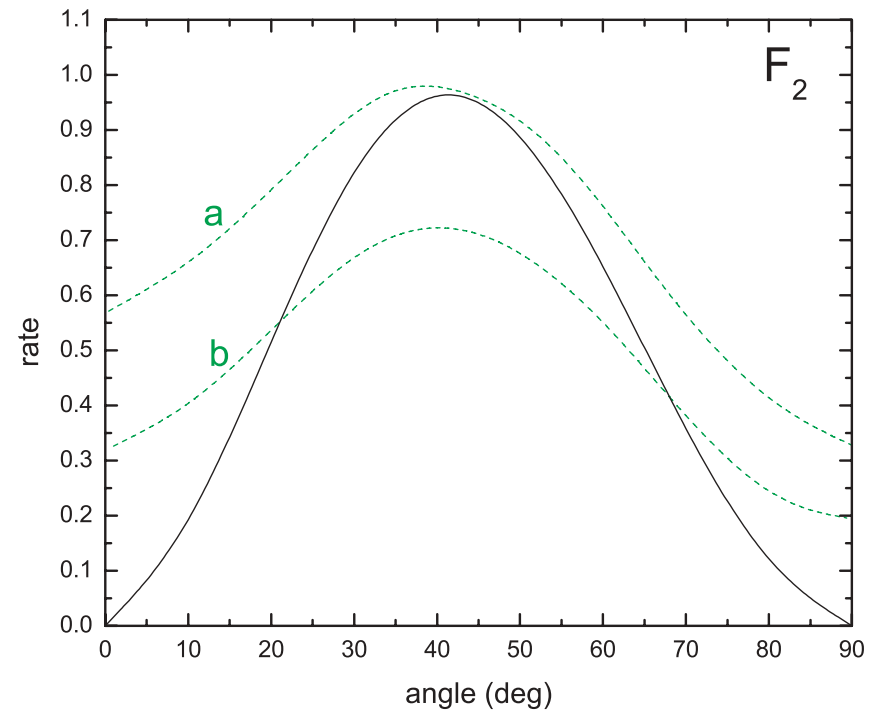

FIG. 11. (Color online) Solid curve, arbitrarily normalized ionization rate for $\mathrm{F}_{2}$ as a function of $\theta_{F}$ for intensity $2 \times 10^{14} \mathrm{~W} / \mathrm{cm}^{2}$. Dashed curves, calculations of Telnov and Chu [21] for total ionization probability (curve a) and the HOMO contribution (curve b). Dashed curves are normalized arbitrarily relative to the solid curve, but their ratio corresponds to that given in Ref. [21].

dependence of the ionization rate becomes stronger, a relationship inconsistent with MO-ADK results [35].

In Fig. 11 we present the angular distribution for $\mathrm{F}_{2}$ and compare it with the results of time-dependent density-functional calculations of Telnov and Chu [21]. As in the case of $\mathrm{N}_{2}$ they found a substantial contribution of HOMO-1 to the total ionization probability. However, in contrast to the $\mathrm{N}_{2}$ case, the shape of the curve representing the total ionization probability is the same as that giving the ionization of HOMO. Both give substantial contributions at $\theta_{F}=0$, in contrast to our result.

In Fig. 12 we present the angular distribution for the $\mathrm{CO}_{2}$ molecule. In comparison with experiment, our calculation suffers from the same drawback as others [22,23,37,45]: the calculated angular distribution is too broad. Moreover, although the peak angle increases with intensity, it is too low compared to the experimental value: at $I=1.1 \times 10^{14} \mathrm{~W} / \mathrm{cm}^{2}$ the calculated distribution peaks at $\theta_{F}=28^{\circ}$, whereas the experimental value is $46^{\circ}$. On the other hand, our distribution is substantially narrower than those produced in other calculations including the strong-field approximation [45] and the TDSE method [22,23]. In particular, as in the case of $\mathrm{O}_{2}$, our ionization rate goes to 0 at $\theta_{F}=0$ and $90^{\circ}$ in agreement with experiment. It is surprising that even TDSE calculations which are supposed to incorporate the correct symmetry of molecular orbitals do not yield low values of ionization rate at $\theta_{F}=0$ and $90^{\circ}$. We note that, in contrast, the TDSE results of Kamta and Bandrauk [18] clearly show nodal planes in ionization of $\sigma_{u}$ and $\pi_{g}$ orbitals of $\mathrm{H}_{2}^{+}$.

Zhao et al. [37] suggested that the experiment of Pavičić et al. [34] should be reexamined. They compared their $\mathrm{CO}_{2}$ data with the experiment of Thomann et al. [46] which agrees much better with MO-ADK and TDSE calculations. Although there are some experimental issues, we also think

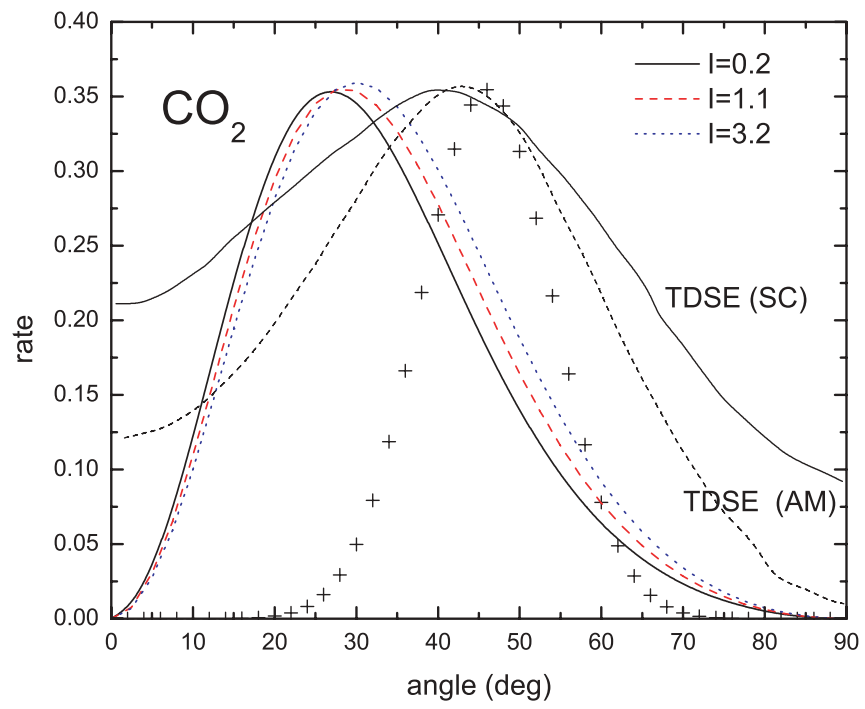

FIG. 12. (Color online) The $\mathrm{CO}_{2}$ ionization rate as a function of $\theta_{F}$, normalized to 0.3546 at $\theta_{F}=27.5^{\circ}$, for three values of intensity. Crosses, experiment [34] at $I=1.3 \times 10^{14} \mathrm{~W} / \mathrm{cm}^{2}$, normalized to 0.3546 at $\theta_{F}=46^{\circ}$. The curves TDSE represent TDSE calculations of Son and Chu [23] (SC, solid) and Abu-samha and Madsen [22] (AM, dashed), as quoted in Ref. [37], normalized to 0.3546 at the peak.

that the theoretical approaches should be developed further. On one hand, we should understand why the TDSE calculations $[22,23]$ produce values of the ionization rate at $\theta_{F}=0$ and $90^{\circ}$ which are significantly higher than ours. On the other hand, the semiclassical method requires further development, in particular in the above-barrier ionization region.

\section{CONCLUSION}

The semiclassical complex time calculations for diatomic molecules show that, like in the case of the atomic systems discussed in Ref. [24], the ADK theory typically overestimates the tunneling ionization rate by a factor of 2 to 3 , but for comparison purposes one has to make sure that the molecular orbitals used in both calculations are the same. Most of the cases of molecular suppression can be explained by the present method; however, the case of ionization of $\mathrm{F}_{2}$ versus Ar is still in some disagreement with experiment.

The situation with $\mathrm{F}_{2}$ is puzzling. Usachenko et al. [31], unlike the present study, which used the quantum chemical methods described in Sec. IIF, determined the initial state and its orbitals with DFT theory. Their treatment depended on the LB- $\alpha$ correlation functional of van Leeuwen and Baerends [47], which was constructed with the intent of better treating molecular bond dissociation energies. Usachenko et al. attribute their success with $\mathrm{F}_{2}$ to the calculated electron correlation given to their representation of the initial state of the molecule by using the LB- $\alpha$ functional. We have no access to this DFT variant, but more popular hybrid functionals, such as B3LYP, with our basis give HOMOs only slightly different from our $a b$ initio ones.

Our results when calculating the dependence of the ionization rate on the angle $\theta_{F}$ between the molecular axis and the field direction agree quite well with experiments for $\mathrm{N}_{2}$ and $\mathrm{O}_{2}$ molecules. However, disagreement between experimental and 
theoretical results for $\mathrm{CO}_{2}$ remains unexplained. In particular the peak of the ionization rate occurs at a much lower angle than observed experimentally. At the same time our calculations demonstrate the suppression of ionization rates for $\theta_{F}=0$ and $90^{\circ}$ in agreement with experiment. The MO-ADK theory and calculations based on numerical integration of TDSE also exhibit suppression of the ionization rate at $\theta_{F}=0$ and $90^{\circ}$ for $\mathrm{O}_{2}$ and $\mathrm{CO}_{2}$ molecules, but not as deep as in the present calculations.

For a clearer understanding of the problem the semiclassical theory should be developed further. In particular it should be extended to the barrier-suppression regime where the classical ionization becomes possible. In this region the theory in fact becomes even simpler, since it does not require an extension of classical trajectories into the complex time domain.

\section{ACKNOWLEDGMENTS}

The authors are grateful to C. D. Lin for helpful comments on the MO-ADK calculations and to D. M. Villeneuve for providing experimental data from Ref. [34] in numerical form. This work was supported by the National Science Foundation under Grant No. PHY-0652866.

\section{APPENDIX: THE CURRENT FOR MANY-PARTICLE DECAY}

Let us start with the Schrödinger equation for an $N$-particle wave function $\Psi\left(\mathbf{r}_{1}, \mathbf{r}_{2}, \ldots\right)$

$$
i \hbar \frac{\partial \Psi}{\partial t}=-\frac{\hbar^{2}}{2 m} \nabla^{2} \Psi+V \Psi
$$

where

$$
\nabla^{2}=\nabla_{1}^{2}+\nabla_{2}^{2}+\cdots
$$

Following the standard procedure, we obtain

$$
\frac{\partial}{\partial t}\left(\Psi^{*} \Psi\right)+\frac{\hbar}{m} \operatorname{Im} \nabla \cdot\left(\Psi^{*} \nabla \Psi\right)=0,
$$

where

$$
\nabla=\nabla_{1}+\nabla_{2}+\cdots
$$

Integrating equation (A2) over $3 N$ dimensional volume $V$, we obtain the conservation of particles law in the form

$$
\frac{d N}{d t}+\frac{\hbar}{m} \int d \mathbf{r}_{1} d \mathbf{r}_{2} \cdots \nabla \cdot \operatorname{Im}\left(\Psi^{*} \nabla \Psi\right)=0,
$$

where $N$ is the number of particles in $V$.
Using the divergence theorem in $3 N$-dim space, we convert the volume integral into the integral over a rectangular hypersurface defined by the equations

$$
z_{1}=a_{1}, \quad z_{2}=a_{2}, \ldots
$$

The integral over this hypersurface can be split into the sum of integrals over each side of the hypersurface with the result

$$
\begin{aligned}
\int & d \mathbf{r}_{1} d \mathbf{r}_{2} \cdots \nabla \cdot\left(\Psi^{*} \nabla \Psi\right) \\
= & \int d \mathbf{r}_{2} d \mathbf{r}_{3} \cdots d \mathbf{S}_{1} \cdot\left(\Psi^{*} \nabla \Psi\right) \\
& +\int d \mathbf{r}_{1} d \mathbf{r}_{3} \cdots d \mathbf{S}_{2} \cdot\left(\Psi^{*} \nabla \Psi\right)+\cdots
\end{aligned}
$$

where $d \mathbf{S}_{1}, d \mathbf{S}_{2} \ldots$, are surface elements in corresponding 3-dim spaces. Therefore the total current through the hypersurface $z_{1}=a_{1}, z_{2}=a_{2}, \ldots$ can be defined as

$$
I=\int_{z_{1}=a_{1}} \mathbf{J}\left(\mathbf{r}_{1}\right) \cdot d \mathbf{S}_{1}+\int_{z_{2}=a_{2}} \mathbf{J}\left(\mathbf{r}_{2}\right) \cdot d \mathbf{S}_{2}+\cdots,
$$

where the current density through the hypersurface $z_{1}=a_{1}$ is given by

$$
\mathbf{J}\left(\mathbf{r}_{1}\right)=\left.\frac{\hbar}{m} \int d \mathbf{r}_{2} d \mathbf{r}_{3} \cdots \operatorname{Im}\left(\Psi^{*} \nabla \Psi\right)\right|_{z_{1}=a_{1}} .
$$

Suppose now that we have a two-electron atom in a static field. In the independent-particle model

$$
\Psi\left(\mathbf{r}_{1}, \mathbf{r}_{2}\right)=\psi\left(\mathbf{r}_{1}\right) \psi\left(\mathbf{r}_{2}\right) .
$$

Both factors have an outgoing flux at $z_{1} \rightarrow \infty$ or $z_{2} \rightarrow \infty$, therefore

$$
\Psi^{*} \nabla \Psi=\psi^{*}\left(\mathbf{r}_{1}\right) \psi^{*}\left(\mathbf{r}_{2}\right)\left[\nabla_{1} \psi\left(\mathbf{r}_{1}\right) \psi\left(\mathbf{r}_{2}\right)+\psi\left(\mathbf{r}_{1}\right) \nabla_{2} \psi\left(\mathbf{r}_{2}\right)\right] .
$$

The first term gives nonzero contribution to the integral in $d \mathbf{r}_{2} d \mathbf{S}_{1}$, and the second term to the integral in $d \mathbf{r}_{1} d \mathbf{S}_{2}$. Both integrals are equal, therefore for two equivalent electrons the independent-particle model tells us that the single-particle decay rate should be multiplied by 2 . This conclusion is based on the assumption that a sufficiently accurate total wave function can be constructed from paired one electron orbital. This is reasonable for neutral systems, such as $\mathrm{He}$ and $\mathrm{F}_{2}$, while being inappropriate for an ion like $\mathrm{H}^{-}$. This conclusion is in accord with the similar conclusion in the photoionization problem [48].
[1] L. V. Keldysh, Zh. Eksp. Teor. Fiz. 47, 1945 (1964) [Sov. Phys. JETP 20, 1307 (1965)].

[2] P. B. Corkum, Phys. Rev. Lett. 71, 1994 (1993).

[3] B. M. Smirnov and M. I. Chibisov, Zh. Eksp. Teor. Fiz. 49, 841 (1965) [Sov. Phys. JETP 22, 585 (1966)].

[4] A. M. Perelomov, V. S. Popov, and M. V. Terent'ev, Zh. Eksp. Teor. Fiz. 50, 1393 (1966) [Sov. Phys. JETP 23, 924 (1966)].

[5] M. V. Ammosov, N. B. Delone, and V. P. Krainov, Zh. Eksp. Teor. Fiz. 91, 2008 (1986) [Sov. Phys. JETP 64, 1191 (1986)].
[6] X. M. Tong, Z. X. Zhao, and C. D. Lin, Phys. Rev. A 66, 033402 (2002).

[7] V. P. Krainov and B. Shokri, Zh. Eksp. Teor. Fiz. 107, 1180 (1995) [JETP 80, 657 (1995)]; V. P. Krainov, J. Opt. Soc. Am. B 14, 425 (1997).

[8] D. Bauer and P. Mulser, Phys. Rev. A 59, 569 (1999).

[9] X. M. Tong and C. D. Lin, J. Phys. B 38, 2593 (2005).

[10] A. Jaroń-Becker, A. Becker, and F. H. M. Faisal, Phys. Rev. A 69, 023410 (2004).

[11] T. K. Kjeldsen and L. B. Madsen, J. Phys. B 37, 2033 (2004). 
[12] T. K. Kjeldsen and L. B. Madsen, Phys. Rev. A 71, 023411 (2005).

[13] V. I. Usachenko and Shih-I. Chu, Phys. Rev. A 71, 063410 (2005).

[14] T. K. Kjeldsen and L. B. Madsen, Phys. Rev. A 73, 047401 (2006).

[15] V. I. Usachenko, Phys. Rev. A 73, 047402 (2006).

[16] X. Chu and Shih-I. Chu, Phys. Rev. A 63, 013414 (2000).

[17] G. L. Kamta and A. D. Bandrauk, Phys. Rev. A 71, 053407 (2005).

[18] G. Lagmago Kamta and A. D. Bandrauk, Phys. Rev. A 74, 033415 (2006).

[19] X. Chu and Shih-I. Chu, Phys. Rev. A 70, 061402(R) (2004).

[20] D. A. Telnov and Shih-I. Chu, Phys. Rev. A 79, 041401(R) (2009).

[21] D. A. Telnov and Shih-I. Chu, Phys. Rev. A 80, 043412 (2009).

[22] M. Abu-samha and L. B. Madsen, Phys. Rev. A 80, 023401 (2009).

[23] S. K. Son and Shih-I. Chu, Phys. Rev. A 80, 011403(R) (2009).

[24] I. I. Fabrikant and G. A. Gallup, Phys. Rev. A 79, 013406 (2009).

[25] T. Brabec, M. Côté, P. Boulanger, and L. Ramunno, Phys. Rev. Lett. 95, 073001 (2005).

[26] V. S. Popov, Yad. Fiz. 68, 717 (2005) [Phys. At. Nucl. 68, 686 (2005)].

[27] A. Talebpour, C.-Y. Chien, and S. L. Chin, J. Phys. B 29, L677 (1996).

[28] C. Guo, M. Li, J. P. Nibarger, and G. N. Gibson, Phys. Rev. A 58, R4271 (1998).

[29] E. Wells, M. J. DeWitt, and R. R. Jones, Phys. Rev. A 66, 013409 (2002).

[30] J. Muth-Böhm, A. Becker, and F. H. M. Faisal, Phys. Rev. Lett. 85, 2280 (2000).

[31] V. I. Usachenko, P. E. Pyak, and V. V. Kim, Phys. Rev. A 79, 023415 (2009).
[32] D. Pinkham and R. R. Jones, Phys. Rev. A 72, 023418 (2005).

[33] I. V. Litvinyuk, K. F. Lee, P. W. Dooley, D. M. Rayner, D. M. Villeneuve, and P. B. Corkum, Phys. Rev. Lett. 90, 233003 (2003).

[34] D. Pavičić, K. F. Lee, D. M. Rayner, P. B. Corkum, and D. M. Villeneuve, Phys. Rev. Lett. 98, 243001 (2007).

[35] Z. X. Zhao, X. M. Tong, and C. D. Lin, Phys. Rev. A 67, 043404 (2003).

[36] A.-T. Le, X. M. Tong, and C. D. Lin, J. Mod. Opt. 54, 967 (2007).

[37] S.-F. Zhao, C. Jin, A.-T. Le, T. F. Jiang, and C. D. Lin, Phys. Rev. A 80, 051402(R) (2009).

[38] J. B. Delos, Adv. Chem. Phys. 65, 161 (1986).

[39] M. W. Schmidt, K. K. Baldridge, J. A. Boatz, S. T. Elbert, M. S. Gordon, J. H. Jensen, S. Koseki, N. Matsunaga, K. A. Nguyen, S. J. Su, T. L. Windus, M. Dupuis, and J. A. Montgomery, J. Comput. Chem. 14, 1347 (1993).

[40] G. A. Gallup, R. L. Vance, J. R. Collins, and J. M. Norbeck, Adv. Quantum Chem. 16, 229 (1982).

[41] A. Saenz, J. Phys. B 33, 4365 (2000).

[42] M. J. DeWitt, E. Wells, and R. R. Jones, Phys. Rev. Lett. 87, 153001 (2001).

[43] M. J. DeWitt and R. J. Levis, J. Chem. Phys. 108, 7739 (1998).

[44] The angular distributions published in Ref. [35] contain some numerical errors. We are grateful to C. D. Lin for bringing this matter to our attention.

[45] V. H. Le, N. T. Nguyen, C. Jin, A. T. Le, and C. D. Lin, J. Phys. B 41, 085603 (2008).

[46] I. Thomann, R. Lock, V. Sharma, E. Gagnon, S. T. Pratt, H. C. Kapteyn, M. M. Murnane, and W. Li, J. Phys. Chem. A 112, 9382 (2008).

[47] R. van Leeuwen and E. J. Baerends, Phys. Rev. A 51, 170 (1995).

[48] K. Krajewska, I. I. Fabrikant, and A. F. Starace, Phys. Rev. A 74, 053407 (2006). 